Traduire le nom des personnages de Love's Labour's Lost : ethnocentrisme et exotisme dans une pièce cosmopolite

Laetitia Sansonetti

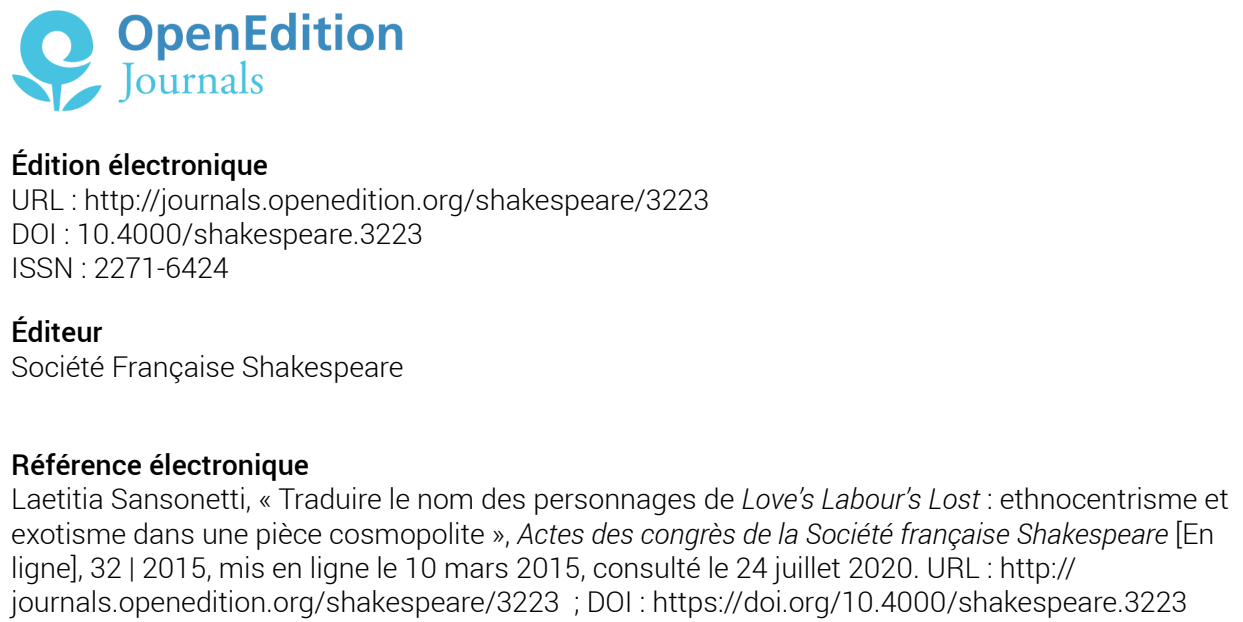

Société Française Shakespeare

Référence électronique

Laetitia Sansonetti, «Traduire le nom des personnages de Love's Labour's Lost : ethnocentrisme et exotisme dans une pièce cosmopolite », Actes des congrès de la Société française Shakespeare [En ligne], 32 | 2015, mis en ligne le 10 mars 2015, consulté le 24 juillet 2020. URL : http:// journals.openedition.org/shakespeare/3223; DOI : https://doi.org/10.4000/shakespeare.3223

Ce document a été généré automatiquement le 24 juillet 2020.

(C) SFS 


\title{
Traduire le nom des personnages de Love's Labour's Lost : ethnocentrisme et exotisme dans une pièce cosmopolite
}

\author{
Laetitia Sansonetti
}

\section{Entre le même et l'autre, penser l'identité}

\section{La traduction comme répétition}

\author{
ROSALINE. What would these strangers? Know their minds, Boyet. \\ If they do speak our language, 'tis our will \\ That some plain man recount their purposes. \\ Know what they would? \\ BOYET. What would you with the princess? \\ BEROWNE. Nothing but peace and gentle visitation. \\ ROSALINE. What would they, say they? \\ BOYET. Nothing but peace and gentle visitation. \\ ROSALINE. Why, that they have, and bid them so be gone. \\ BOYET. She says, you have it and you may be gone ${ }^{1}$.
}

Lors de leur piteuse " ambassade ", les hommes de Navarre déguisés en Moscovites sont accueillis par un intermédiaire, Boyet, qui sert de traducteur entre ces pseudoinconnus et les dames de France, bien décidées à saboter le divertissement. Mais le processus de traduction, c'est-à-dire de transmission d'information par le transfert d'une langue à l'autre, s'apparente bien plutôt à la répétition comique qu'à un moyen de communication. Bien que rien dans la graphie ne le laisse supposer, il est possible d'imaginer que les Navarrais aient adopté un accent pour déguiser leur voix comme ils ont masqué leur identité d'un costume exotique. Mais il est tout aussi possible de se délecter de la répétition quasi à l'identique, le passage du discours direct au discours 
indirect permettant de souligner les enjeux de pouvoir et de représentation, c'est-àdire à la fois d'image projetée et de délégation.

1 Un peu plus loin dans la scène du Masque, le jeu de mots de Katharine sur la seconde moitié du nom de Longaville pourrait se comprendre comme une allusion à l'accent "étranger " qu'il chercherait à adopter: "'Veal', quoth the Dutchman. Is not veal a calf?» (v.ii.247). En tout cas, ce « veal » renvoie à l'altérité supposée de ce soupirant qui, se croyant anonyme, courtise la mauvaise personne, en jouant non seulement sur la langue, mais aussi sur les langues, puisque la plaisanterie ne se comprend qu'en imaginant à la fois une prononciation défectueuse de «well » et l'origine française du terme qui désigne la viande de veau. Le décalage introduit par la prononciation crée également un décalage au sein même de l'anglais, puisque la corruption phonétique de «well» à «veal» passe par "veil», mot qui renvoie au déguisement des deux personnages concernés ${ }^{2}$.

2 Les deux scènes de traduction orale quasi simultanée que je viens de mentionner montrent que le processus de traduction se rapporte à des enjeux thématiques et dramatiques essentiels pour la pièce, à savoir l'expression (physique et linguistique) de l'identité et la difficulté d'une communication efficace (sans oublier les délices comiques que génèrent les échecs de communication ${ }^{3}$ ).

3 Ma réflexion sur la traduction des noms propres abordera donc la dimension interlinguistique du questionnement sur l'identité sous deux aspects qui se font écho: au sein de la pièce, la forme paradoxale de cosmopolitisme qui se dégage de la mise en présence de personnages d'origines disparates; dans les traductions en français de la pièce depuis Le Tourneur au dix-huitième siècle jusqu'à la version la plus récente de Jean-Michel Déprats, la tension entre deux voies possibles pour la traduction, l'ethnocentrisme et l'exotisme.

\section{Ethnocentrisme et exotisme}

4 Le terme d'« ethnocentrisme » apparaît pour la première fois dans les travaux du sociologue américain William Graham Sumner en 1906: «Ethnocentrism is the technical name for this view of things in which one's own group is the center of everything, and all others are scaled and rated with reference to it ${ }^{4}$. " L'attitude qu'il désigne se retrouve dans tous les groupes humains depuis l'origine de l'humanité, ainsi qu'en témoigne la liste des exemples fournis par Sumner ; l'un des plus connus, et des plus intéressants du point de vue linguistique, est celui des Grecs, qui appelaient «barbares » les étrangers, mimant par la désignation onomatopéique ("bárbaros») la différence linguistique qui motivait l'exclusion.

5 Quant à l'exotisme, il part lui aussi de la perception de la différence comme altérité, faisant de l'autre un objet de curiosité à cause de sa distance (géographique, culturelle, symbolique ${ }^{5}$ ). Or, c'est justement à la Renaissance que les contacts entre différents systèmes culturels éloignés géographiquement se développent et se renforcent à la faveur des voyages vers des contrées inconnues jusqu'alors, d'où les voyageurs ramènent des images et des récits qui vont susciter la fascination et mettre en branle l'imagination. Et c'est à la fin du seizième siècle qu'apparait le terme en anglais ${ }^{6}$.

6 En traduction, Henri Meschonnic appelle "ethnocentrisme " une "tradition " qu'il dénonce parce qu'elle « ramène l'autre au même » en accomplissant « l'effacement de l'altérité ${ }^{7}$ ", c'est-à-dire qu'elle vise à donner l'impression, l'illusion que le texte dit 
"source" a été écrit dans la langue dite "cible». Meschonnic prône plutôt une conception de la traduction qui soit « une transformation de l'identité par la diversité 8.» Dans le même ordre d'idées, Antoine Berman affirme que "l'essence de la traduction est d'être ouverture, dialogue, métissage, décentrement ${ }^{9}$ " et souhaite en finir avec la traduction "ethnocentrique ${ }^{10}$ ». Réduire l'Autre au Même en l'assimilant (s'il le faut par la force) ou l'exclure (par la ségrégation); utiliser l'altérité pour penser l'identité. Ces deux attitudes ne se laissent jamais aussi clairement percevoir que dans l'utilisation de la langue.

\section{Une pièce cosmopolite?}

\section{Nomen est numen}

7 L'article de Ladan Niayesh dans le présent recueil analyse en détail les fonctions de l'étranger et des étrangers dans la pièce. Je lui emprunterai mon premier exemple, celui du nom de Berowne. L'orthographe n'est pas sans évoquer la couleur brune, ce qui l'inscrit dans le réseau de l'altérité sombre auquel sont également associés la brune Rosaline (malgré son nom) et l'Espagnol Don Adriano de Armado, originaire d'un pays associé à la couleur bistre («tawny Spain» [I.i.171]). Dans un article sur les mots étrangers dans le théâtre de Shakespeare, Mylène Lacroix attire l'attention sur le titre même de Don Armado, "que Costard s'empresse de corrompre en Dun Adramadio [Iv.iii.190], se moquant ainsi, à son insu, du teint basané de l'Espagnol ${ }^{11}$ », puisque l'adjectif « dun » renvoie à la couleur brune. Nomen est numen donc, le nom dit l'essence, mais par le truchement d'un jeu sur les sonorités qui nécessite une conversion translinguistique.

8 La confusion phonétique de Costard va plus loin, puisqu'elle renforce l'association au monde guerrier en ajoutant à la référence espagnole à l'homme armé (armado), ou à l' Armada de Philippe II, l'écho du tambour martial (drum). On pourrait aussi y entendre l'expression sonore de son association à Moth à travers dram, la drachme, mesure équivalant à un seizième d'once et renvoyant donc à une quantité négligeable. Mais on y entend surtout une succession de consonnes précipitées ; il est bien difficile de jeter la pierre à ce pauvre Costard, car prononcer -DRN- suivi de -RMD- est un défi digne de Démosthène, l'orateur grec qui avait surmonté son bégaiement en s'entraînant à déclamer la bouche pleine de galets. Dull, lui, n'arrive même pas à prononcer le nom du Signeour (I.i.184 $\left.4^{12}\right)$.

9 Si la faconde maniérée d'Armado l'expose aux moqueries, son langage, qui repose sur l'utilisation de certains archaïsmes poétiques saxons (comme "yclept» [I.i.228]) et la création d'adjectifs composés, n'évoque pas vraiment l'Espagne. Quand il utilise une expression espagnole (qui pourrait tout aussi bien être italienne), non seulement c'est un cliché emprunté à Sénèque, mais il la met à distance en employant la troisième personne du pluriel : «we will put it, as they say, to fortuna de la guerra» $\left(\right.$ v.ii.524 $\left.{ }^{13}\right)$. Ainsi que le souligne Lynn Magnusson,

However much [Armado's] name may seem reminiscent of an event associated with hatred of the Spanish, there is nothing of the threatening or despised alien, even if he is a figure of fun [...]. [H] comes to be cast as an aspiring immigrant, eager to assimilate ${ }^{14}$. 
Le vocabulaire de l'acculturation (« immigrant», "assimilate») signale la transition d'une utilisation « exotique » du personnage à un point de vue "ethnocentrique ». Cet Espagnol qui linguistiquement n'a que peu d'Espagne en lui si ce n'est son nom et l'insistance des autres personnages à mentionner son pays d'origine, faut-il néanmoins l'imaginer parlant avec un accent espagnol ? Je reviendrai sur cette question dans ma dernière section.

Retournons à Berowne, personnage qui pourrait évoquer Armand de Gontaut, baron de Biron, ou son fils Charles, duc de Biron, tous deux liés à Henri IV le Navarrais. Tout comme ses comparses Longaville et Dumaine, Berowne porte un nom à consonance française orthographié à l'anglaise ${ }^{15}$. La transcription sert donc ici à assurer une prononciation à peu près conforme à la prononciation française, ce qui rend ces personnages à la fois plus «anglais" dans la graphie et plus "français » dans les sonorités.

11 Les noms des compagnes de la princesse française, qui ne sont que des prénoms, sont quant à eux repérés par la critique au sein du canon shakespearien: Katherine a presque le même nom que Katharina, l'héroïne « italienne » de The Taming of the Shrew, mais rappelle aussi la princesse Katherine de France courtisée par Henry dans Henry V; Maria possède une homonyme dans Twelfth Night; Rosaline fait penser au premier amour de Roméo dans Romeo and Juliet et à la Rosalind d'As You Like It. Les variations de graphie entre ces différentes pièces suffisent à rappeler que le mélange des nationalités et des langues est une caractéristique de l'onomastique shakespearienne.

Quant aux personnages secondaires possédant un nom anglais, ils se singularisent par le fait que leur nom anglais renvoie à un nom ou un adjectif qui reflète leur essence. Ici, pas de référence à une identité géographique mais une utilisation métaphorique du nom, dont le sens doit être compris immédiatement en anglais : Moth est petit et jeune, Costard a une grosse tête, Dull n'est pas très vif d'esprit.

\section{Traduction et identité}

13 Moth n'est toutefois pas "purement » anglais, puisque son nom invite lui aussi au jeu de mots interlinguistique. Rapprochant "moth» du français «mot », rapprochement adéquat si l'on pense à la vivacité langagière du petit page, W.C. Carroll définit ainsi la pièce : "a 'French' play for a courtly (French-speaking?) audience ${ }^{16}$.» La corrélation entre la dimension sociale et la dimension cosmopolite, ou du moins la capacité à évoluer dans un milieu bilingue anglais-français, comme l'a été pendant très longtemps la Cour anglaise, voire plurilingue, comme elle l'était sans doute à l'époque d'Élisabeth, me semble très intéressante.

14 Le jeu de mots de Katherine précédemment cité mobilise le français, l'anglais et le néerlandais en lien avec la réflexion sur l'identité et l'altérité géographique; d'autres jeux de mots mettent en lumière le rôle joué par la traduction dans l'établissement d'une hiérarchie sociale en rapport avec l'aliénation linguistique. Le terme "brawl», employé par Moth pour suggérer à Armado un moyen de conquérir sa bien-aimée, est mal compris par son interlocuteur, en vertu de l'homophonie entre le mot désignant la danse de cour lente, importé du français «branle», et le mot à l'origine incertaine désignant une rixe bruyante ${ }^{17}$ : 


\begin{abstract}
MOTH. Master, will you win your love with a French brawl?
ARMADO. How meanest thou? Brawling in French?

мотн. No, my complete master; but to jig off a tune at the tongue's end, canary to it with your feet, humour it with turning up your eyelids, sigh a note and sing a note, sometime through the throat, as if you swallowed love with singing love, sometime through the nose, as if you snuffed up love by smelling love; [...] (III.i.6-12)
\end{abstract}

Il me semble que l'antanaclase sur laquelle repose la confusion entre le branle et la querelle braillarde contient non seulement une dimension trans-artistique (danse, musique, peinture), mais aussi trans-culturelle et sociale. Pour Armado, "French brawl » ne peut être quelque chose de raffiné ; est-ce parce qu'en tant qu'étranger aux mœurs moins délicates et à la bourse moins pleine que son langage ne le laisse supposer (lui qui n'a pas de chemise), il n'est pas au fait des dernières danses à la mode à la Cour? Ou bien Shakespeare utilise-t-il une figure d'étranger pour se moquer du pays qui est apparu pendant des siècles comme « l'Autre » de l'Angleterre, son ennemi intime, insinuant que les Français ne seraient bons qu'à se quereller bruyamment ${ }^{18}$ ? Lorsqu'il chante, Moth le fait en reprenant un air français, "Quand Colinelle... », ou peut-être s'agit-il d'un air irlandais, « Can cailin gheal » (III.i.2) ? Poésie pastorale venue d'une terre autrefois anglaise ou chanson empruntée à un peuple qui refuse de se faire domestiquer par les colons anglais, les figures de l'altérité linguistique ne se laissent assimiler phonétiquement que pour mieux affirmer leur étrangeté ${ }^{19}$.

La dimension sociale du jeu de mots polyglotte est particulièrement manifeste dans les dialogues entre les pédants et les rustres. Pour savourer ces jeux de mots, il faut mesurer le décalage entre les locuteurs qui se targuent de dominer le latin et ceux dont la maitrise de la langue anglaise est approximative. Patricia Parker en cite plusieurs, dont le latin "haud credo » de Holofernes: le terme n'est pas compris par Dull, qui se permet de corriger le pédant dans son identification erronée de l'animal tué lors de la partie de chasse («it was a pricket » [Iv.ii.18 $\left.{ }^{20}\right]$ ). Le même contraste est à l'œuvre entre l'impératif latin invitant Holofernes à poursuivre la démonstration de son talent de poète, «Perge » (Iv.ii.48), et son homophone la " purge » scatologique qu'évoquent des mots tels que « pollution » et « scurrility ${ }^{21} »($ Iv.ii.42 et 49).

\title{
Traduire le nom des personnages
}

17 Les traducteurs français de la pièce ont eu recours à des stratégies qui reflètent des rapports différents aux deux pôles de l'ethnocentrisme et de l'exotisme (ou défamiliarisation) définis précédemment. Je distinguerai trois groupes de personnages, les «Navarro-français ", les "Anglais » et les "Italespagnols », dont j'étudierai les noms dans les traductions de Pierre Le Tourneur (1782), François-Victor Hugo (1860), Émile Montégut (1867), Pierre Messiaen (1939), Jean-Louis Supervielle (1955), Annie Berthet (1969), Jean Malaplate (2000) et Jean-Michel Déprats $(1990,2013)^{22}$.

\section{La scène est en Navarre}

Puisque les hommes de Navarre tiennent leur nom de personnages historiques ayant appartenu au cercle proche ou lointain d'Henri IV, les traducteurs dans leur grande majorité leur donnent une orthographe française: Berowne devient «Biron ${ }^{23}$ ", 
Longaville est écrit "Longueville » et Dumaine est parfois scindé en deux. Seul JeanMichel Déprats se démarque de ce consensus en choisissant de conserver "Berowne ", graphie qui défamiliarise le gentilhomme navarrais (ou périgourdin, puisque telle était l'origine de la famille de Biron). Dans les notes à l'édition bilingue de la Pléiade, il justifie ainsi ce choix :

Le nom de Berowne est si attaché au rôle d'aristocrate persifleur de Peines d'amour perdues, et si peu en rapport avec l'ombrageux maréchal qu'évoquera Chapman [dans La Conspiration et la Tragédie de Charles, duc de Biron, que George Chapman orthographie Byron, en 1608], qu'il a été conservé dans la présente traduction pour le distinguer de tout personnage historique contemporain ${ }^{24}$.

Pour Déprats, il s'agit de ne pas mettre sur la fausse piste d'une pièce "à clef » historique afin de ne pas parasiter la lecture.

Dans son approche des noms propres français, le traducteur français est ainsi confronté à un dilemme : s'il conserve la graphie d'origine, il transforme un effet d'assimilation (l'écriture phonétique « à l'anglaise » de noms étrangers) en défamiliarisation (difficile de reconnaître en Berowne un Navarrais - c'est bien la raison pour laquelle Déprats conserve "Berowne", dont le nom ne fait référence qu'au personnage créé par Shakespeare et n'a donc de sens qu'au sein de la pièce); s'il donne à tous les personnages un nom à consonance française, il risque de tomber dans l'acculturation forcée, l'assimilation indiscriminée.

Deux prénoms d'origine biblique méritent également notre attention. L'équivalent français de Nathaniel, "Nathanaël ${ }^{25}$ ", est choisi par Supervielle, Berthet et Malaplate, les autres préférant conserver la graphie anglaise. Le nom du pédant Holofernes, qui est orthographié avec un -f- dans les premières éditions, renvoie lui aussi à une figure biblique et s'écrit traditionnellement avec -ph-. L'équivalent français n'a pas de -s à la fin, ce qui ne semble pas poser de problème à la traduction. Pourtant, choisir «Holopherne » plutôt que «Holof/phernes », c'est renvoyer explicitement, dans son orthographe moderne, au personnage biblique décapité par Judith d'après le « Livre de Judith ", texte deutérocanonique, et donc attirer l'attention sur l'origine extradramatique du pédant.

Messiaen propose une version française sans -s mais avec un -f- intervocalique, choix qui me semble refléter une volonté de ne pas assimiler ce qui relève d'un exotisme non plus géographique mais temporel. En effet, il est connu que le pédant de Shakespeare trouve un ancêtre chez le précepteur de Gargantua, "un grand docteur en theologie nommé maistre Thubal Holoferne, qui luy aprint sa charte si bien qu'il la disoit par cueur au rebours ${ }^{26}$. $"$ L'orthographe française n'étant pas plus fixée à l'époque de Rabelais (première moitié du seizième siècle) que ne l'était l'orthographe anglaise du temps de Shakespeare, surtout pour la translittération de mots d'origine hébraïque, le nom qui s'écrit de nos jours "Holopherne " pour souligner son origine "étrangère » (puisque -ph- et -f- se prononcent de la même manière) s'écrivait « Holoferne » il y a cinq cents ans. Choisir "Holoferne" plutôt que "Holopherne ", c'est renvoyer à Rabelais explicitement, non seulement en tant que texte-source, mais en tant que texte du seizième siècle : c'est défamiliariser sur le mode temporel. 


\section{Les jeux de mots en anglais} pour les traducteurs, défi que seul Le Tourneur n'a pas relevé, laissant tels quels les noms de Dull, Costard et Moth ${ }^{27}$. On pourrait penser que c'est parce qu'il n'a pas compris qu'il y avait là des jeux de mots à transmettre aux lecteurs français. Mais l'appendice qui suit la pièce donne une autre explication, liée à l'évolution du goût : Le Tourneur, comme Samuel Johnson, détestait les jeux de mots, qu'il estimait indignes du génie en général et de celui de Shakespeare en particulier ${ }^{28}$. Il explique ainsi pourquoi il n'a pas traduit le poème sur le cerf tué à la chasse, qu'il inclut malgré tout mais dans une traduction "mot à mot" qu'il estime de toute façon inutile parce que pour comprendre ces jeux de mots ineptes, « il faut savoir l'Anglais » : « Et les équivoques de sore, plaie, avec soare, chevreuil de quatre ans, \& $L$, lettre \& chiffre romain valant cinquante. Tout cela ne vaut pas la peine d'être entendu ${ }^{29}$." Parmi les "Retranchements ", c'est-à-dire les scènes retranchées du corps de la traduction et rejetées à la fin (mais pas complètement effacées, par souci d'honnêteté), figure justement la scène entre Costard, Moth et Armado où apparaît le jeu de mots assurant la mise en rapport du physique de Costard avec son nom : «Un prodige, mon maître! Voici une grosse tête brisée au menton »; la note de bas de page indique : " a costard: équivoque sur ce nom, qui signifie une pomme à grosse tête ${ }^{30}$. Décidément peu sensible au comique décalé de la scène, Le Tourneur semble également avoir confondu «chin» et «shin» (III.i.59), gommant l'aberration physique du contact entre tête et tibia $^{31}$.

Chez les autres traducteurs, "Balourd» l'emporte pour traduire Dull; Berthet en propose une version assez proche, «Lourdaud», tandis que Montégut avait préféré "Lépais »; Supervielle a une trouvaille qui me semble particulièrement réussie, un «Butor» digne d'Edmond Rostand qui est aussi un nom propre attesté (et c'est peutêtre la raison pour laquelle les traducteurs suivants, œuvrant alors que l'homme de lettres français Michel Butor gagnait en célébrité, ne l'ont pas suivi dans cette voie). Quant à Costard, seuls deux traducteurs, Messiaen et Déprats, ont réussi à lui conserver sa double référence, à la tête et à un fruit, en le nommant respectivement « Potiron » et " Courge ". Pour Hugo, il est " Trogne ${ }^{32}$ ", que Berthet altère légèrement, transformant le nom en surnom avec «La Trogne ", tandis que pour Montégut il est "Grossetête ", pour Supervielle «Cabochard» et pour Malaplate «Caboche». Moth, qui peut désigner au sens large un insecte et pas uniquement les mites ou les papillons de nuit (Montégut traduit "Papillon»), devient exotique (et socialement raffiné ?) dans la traduction technique de Hugo et Messiaen: "Phalène », emprunt savant du français au grec qui remonte au seizième siècle alors que mot moth existait déjà en vieil anglais. Supervielle et Berthet choisissent eux aussi un terme plus récent que l'original anglais avec leur "Moustique", dont Malaplate propose une version plus répandue en France, le «Moucheron ${ }^{33}$ ». Seul Déprats se démarque de l'insecte volant pour insister sur la vivacité du page, signalant que la graphie moth renvoyait également à la particule, au corpuscule (mot qui s'écrit de nos jours mote) : son «Puce», plus bref et vif dans ses sonorités, remplit particulièrement bien sa fonction.

Face aux nobles de Navarre, les personnages dont les noms anglais expriment une caractéristique physique ou intellectuelle appartiennent au domaine de l'indigène pour les spectateurs élisabéthains; les traducteurs se sont donc employés à trouver des 
équivalents qui soient tout aussi immédiatement compréhensibles en français ${ }^{34}$, afin de ne pas faire passer ces personnages pour des curiosités exotiques aux yeux et surtout aux oreilles d'un lectorat francophone $\mathrm{e}^{35}$.

\section{La dimension cosmopolite}

S'il est un personnage que son nom identifie comme un étranger, c'est bien Don Adriano de Armado. Lorsque Boyet le décrit à la Princesse de France, Armado est assimilé par annomination (utilisation d'un nom propre comme nom commun) à un personnage bien connu des Élisabéthains, un certain « Monarcho » :

BOYET. This Armado is a Spaniard that keeps here in court,

A phantasime, a Monarcho, and one that makes sport

To the prince and his book-mates. (Iv.i.91-93)

Ce Monarcho dont le nom était devenu synonyme de folie des grandeurs était un Italien fréquentant la cour d'Élisabeth dans les années 1570 et qui se prenait pour le roi du monde, d'où son surnom ${ }^{36}$. Dans l'assimilation d'Armado à Monarcho, il y a donc double annomination, du nom commun italien (monarca) au surnom anglicisé (Monarcho) à l'emploi comme nom commun (a Monarcho). Thomas Churchyard lui consacre une épitaphe en 1580 :

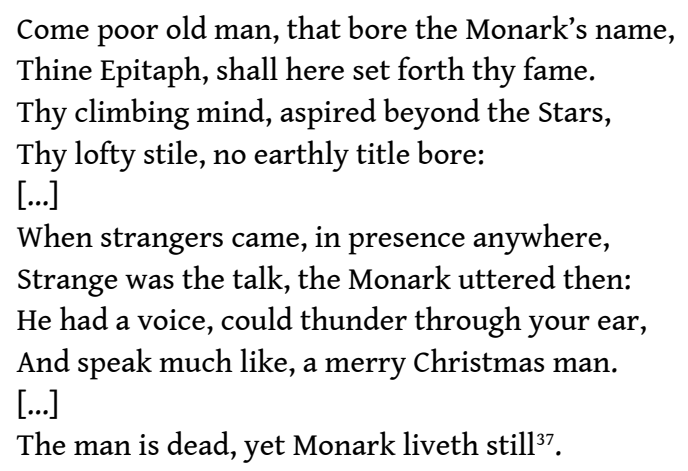

Or, si cet Italien était sans doute bien connu des contemporains de Shakespeare, il n'en va pas de même pour les lecteurs francophones. Puisque la référence originale n'est plus comprise, faut-il adapter ce nom ? La plupart des traducteurs fournissent une note explicative, mais tous ne tentent pas de le traduire, en vertu justement du référent historique. Ceux qui le traduisent, Supervielle et Déprats par «Matamore», Messiaen par "Artaban », ont recours à deux stratégies différentes de déplacement. «Artaban », choisi par Messiaen, est un nom d'origine perse et parthe porté par des princes et des soldats célèbres sur plusieurs siècles dans l'Antiquité; il est connu par l'expression "fier comme Artaban », qui provient d'un roman en treize volumes du dix-septième siècle écrit par Gautier de La Calprenède, intitulé Cléopâtre. La dimension étrangère est certes perdue, malgré l'origine exotique du nom ; toutefois, si comme le disait Boileau à propos de La Calprenède, "Tout a l'humeur Gasconne, en un Autheur Gascon ${ }^{38}$ », cet Armado-Artaban gascon ferait un double parfait pour Biron le Périgourdin.

Quant à Supervielle et Déprats, ils rendent à l'analogue d'Armado une origine méditerranéenne, revenant de l'Italie à l'Espagne. Même si l'équivalence ethnocentrique entre tous les peuples (catholiques) de la Méditerranée semble perdue, le lien entre Espagne et Italie demeure, puisque ce "tueur de Maures» est un 
personnage de la commedia dell'arte, c'est-à-dire la représentation italienne d'un personnage espagnol stéréotypé. Si la référence historique est perdue (et peut-il en être autrement ici ?), l'intertextualité théâtrale est mise en lumière. "Matamore", c'est donc à la fois un nom et une fonction, comme « Monarcho » était à la fois une fonction et un nom, mais le système d'annomination est interne au théâtre, ce qui relance la discussion sur le rapport entre nom et fonction.

\section{Le nom et la fonction}

\section{La fonction plutôt que l'identité} catégorie de personnages stéréotypés héritée de la comédie latine. En effet, Armado est le descendant du miles gloriosus de Plaute ${ }^{39}$, du soldat fanfaron dont on trouve aussi une incarnation dans la lignée des «Capitaines » de la commedia dell'arte italienne, forme théâtrale qui a été rapprochée de Love's Labour's Lost par Daniel Boughner. Même si les deux figures ne sont pas parfaitement superposables, Armado possède plusieurs traits en commun avec le "Capitaine " de la comédie italienne, notamment sa disposition amoureuse, qui le pousse à se confier à son serviteur et le conduit à écrire des lettres enflammées qui n'atteignent pas leur destinataire, créant une confusion cocasse. C'est aussi un voyageur aux aspirations de noblesse qui se retrouve souvent à court d'argent. Mais Armado échappe aux bastonnades que reçoit régulièrement le Capitaine, dont il ne partage pas la passion pour les jeux de dés ou de cartes ni la consommation de vin ${ }^{40}$.

Quant à son hispanité, il la tient aussi de la comédie italienne du seizième siècle, dans laquelle la haine de l'envahisseur colore le type du Capitano pour lui donner des traits espagnols, ainsi que l'explique Boughner : "To create a vehicle of derision, the Italians fused the satire of the Spaniard with the already flourishing role of the captain and set upon the stage the capitano spagnuolo, the supreme exaggeration of the type of the boastful soldier ${ }^{41}$.» françaises de Tabarin, le Capitaine Rodomont ( $\mathrm{du}$ nom du roi d'Alger imaginé par Boiardo dans Roland amoureux [1482] et repris par l'Arioste dans Roland furieux [1516-1532] et qui a donné «rodomontade ») parle un sabir de français, d'italien et d'espagnol :

Cavallierès, mousquetaderes, bombardas, canonés, morions, corseletes! Aqui, veillaco! ... Son il Capitanio Rodomonté, la bravura, la valore de toto del mondo ; la mia spada s'est rendue triomphanté del toto universo. ... Que fasto en sta casa, Tabarin! Que fasto, veillaco? Io te quiero ablar. Aqui, veillacon? Aqui, poerco ? Io te quiero matar, eres moerto ${ }^{42}$ !

Or, on l'a vu, Armado soigne son anglais (jusqu'à l'excès). La comparaison entre la graphie de Tabarin et celle du texte shakespearien (dans laquelle un éventuel accent ne transparaît pas, à la différence des parlers régionaux dans d'autres pièces ${ }^{43}$ ) relance la question de l'identité sonore d'Armado ${ }^{44}$.

31 Christophe Camard rapproche une autre figure interlope du théâtre shakespearien, le Maure vénitien Othello, du soldat fanfaron appelé «Capitaine Spavento " [Épouvante] sur les scènes italiennes : « tout comme cette célèbre figure, Othello est un mercenaire qui raconte ses hauts faits, tombe facilement amoureux et se prétend prince de 
contrées lointaines ${ }^{45}$. »Ce rapprochement me semble d'autant plus intéressant que ces deux soldats sont des figures d'altérité désireuses de s'intégrer, l'exotisme séduisant d'Othello se heurtant à l'ethnocentrisme de ses détracteurs qui l'accusent de n'être qu'un fanfaron. Le basculement de la comédie vers la tragédie survient quand le capitaine beau-parleur et jaloux finit par tuer son épouse innocente, alors qu'Armado ne peut pas se battre en duel pour défendre son honneur et son amour parce qu'il n'a pas chemise : " The naked truth of it is, I have no shirt » (v.ii.686).

L'absence de chemise, justement, le rapproche d'un avatar du soldat fanfaron un peu plus tardif (seconde moitié du dix-septième siècle), le "Capitaine Spezzafer" ("Taillefer »). D'après la pièce des Trois voleurs découverts (Tre ladri scoperti), ce dernier ne portait pas de chemise, ainsi qu'il l'explique à Arlequin :

On prétend, lui dit Arlequin, que vous ne portez point de chemise ?-C'était autrefois ma coutume, lui répond le Capitan, parce qu'alors, comme j'étais extrêmement furieux, aussitôt que je me mettais en colère, le poil, que j'avais abondamment sur tout le corps, se dressait, perçait ma chemise de toutes parts et y faisait tant de trous, qu'on l'aurait prise pour une passoire ; mais depuis quelque temps, m'étant fort modéré, je porte du linge comme les autres.

Et le voilà qui entre dans une « boutique de lingère » pour s'acheter une chemise ${ }^{46}$.

Même si son nom est porteur d'associations que j'ai déjà mentionnées, c'est malgré tout en tant que type qu'Armado est désigné dans la plupart des didascalies et préfixes de discours dans les premières éditions (1598 et 1623) : il est "Braggart ", le vantard. De même Dull est-il «Constable », Costard "Clown », Jaquenetta " Maid» ou « Wench », Holofernes "Pedant» et Moth "Page» ou «Boy ». Le cas des dames françaises est le plus extrême, puisque lors de leur première apparition en I.i, elles sont désignées par des numéros : 1.Lady, 2.Lady et 3.Lady. La première fois qu'elle est nommée, Rosaline semble avoir été confondue avec Katherine. La fonction de chacun des personnages, au détriment de son identité individuelle, est soulignée dans la liste fournie par Berowne à l'approche de la Parade des Neuf Preux : «The pedant, the braggart, the hedge-priest, the fool and the boy » (v.ii.5347).

Étant donné qu'il n'existait pas de liste des personnages au début de la pièce dans les éditions originales, les différentes formules utilisées pour qualifier Armado dans les listes qui font désormais autorité ont été élaborées plus tardivement par les éditeurs à partir d'expressions tirées de la pièce. Leur traduction en français n'entre donc pas dans le cadre de mon étude. En revanche, le choix de Gisèle Venet et Jean-Michel Déprats de conserver la désignation variable dans les préfixes de discours, qui appellent parfois les personnages par leur nom, parfois par leur fonction, mérite d'être remarqué. Ainsi que l'explique Gisèle Venet dans sa notice, "les variations dans les attributions de répliques des principaux personnages types [Armado, Holofernes, Costard, Nathaniel] ont été respectées [...] afin de ne pas gommer la proximité de ces figures avec celles de la commedia dell'arte ${ }^{48} »$.

\section{Le nom est la fonction}

Dans une pièce où triomphent l'annomination et plus généralement les figures de synonymie, ainsi que l'a montré Carroll ${ }^{49}$, le dernier mot ne peut pas revenir à la scission du nom et de la fonction. Le court nom du petit Moth, qui condense déjà une 
polysémie polyglotte explosive puisqu'il est petit insecte, corpuscule, peut-être " mot " (en français dans le texte), ainsi que "page » et "garçon", renvoie également à sa fonction auprès d'Armado. Ainsi que le souligne Boughner dans son article sur la commedia dell'arte, Moth correspond tout à fait au type du parasite: "Armado's relations with the derisive Moth are like those of the boorish master and the jeering servant or parasite of New Comedy. As Moth explains the arts that betray nice wenches, so [...] Gnatho found it necessary to give Thraso elementary instructions in the game of $\operatorname{love}^{50}$.» Or, l'un des sens de moth en anglais, c'est justement celui de parasite. En effet, le terme moth correspond au bas-latin tinea, qui signifie par analogie "personne se comportant comme un parasite ${ }^{51}$.» Moth incarne donc l'idéal d'adéquation entre les mots et les choses, le nom et l'essence, dans sa version théâtrale : le nom est la fonction.

\section{NOTES}

1. William Shakespeare, Love's Labour's Lost, v.ii.175-183, éd. W.C. Carroll, « The New Cambridge Shakespeare ", Cambridge : CUP, 2009. Les citations données ici sont tirées de cette édition.

2. Voir la note de Carroll dans son édition de la pièce, op. cit., ap. loc. Voir également le complément d'information apporté par John Michael Archer, "Love's Labour's Lost ", in A Companion to Shakespeare's Works, vol. III, The Comedies, éds. Richard Dutton et Jean E. Howard, Malden : Blackwell Publishing, 320-337, p. 326 : «Dutch v[e]el also means plenty ».

3. Questions que j'ai abordées dans mon article « Le costume dans Love's Labour's Lost, v.ii : du masque au signe ", Actes des congrès de la Société française Shakespeare 26 (2008), accessible en ligne : http://shakespeare.revues.org/1474 (consulté le 10 février 2015).

4. William Graham Sumner, Folkways, Boston : Ginn and Company, 1906, chapitre I, §15 sqq., p. 13 sqq. Texte accessible en ligne: http://www.gutenberg.org/files/24253/24253-h/24253-h.htm (consulté le 10 février 2015). Incidemment, Sumner donne en épigraphe à son ouvrage trois citations tirées de textes de la Renaissance : une du Courtisan de Baldassare Castiglione et deux de Shakespeare (Hamlet et Coriolan).

5. Voir à ce sujet, entre autres, Tzvetan Todorov, Nous et les autres, La réflexion française sur la diversité humaine, Paris : Seuil, 1989.

6. Voir Oxford English Dictionary, s.v. exotic adj.1, qui cite Ben Jonson, Every Man out of his Humor (1600), IV.iii.30 : « Magique, Witchcraft, or other such Exoticke Artes».

7. Henri Meschonnic, Pour une poétique du traduire, Paris : Verdier, 1999, p. 88-89.

8. Idem, De la langue française, Paris : Hachette, 1997, p. 210.

9. Antoine Berman, L'Épreuve de l'étranger. Culture et traduction dans l'Allemagne romantique, Paris : Gallimard, 1984, p. 16.

10. Idem, La traduction et la lettre, ou, L'auberge du lointain, Paris : Seuil, 1999, p. 29 sqq. En langue anglaise, Lawrence Venuti utilise les termes de "domesticating» (pour la traduction ethnocentriste assimilatrice) et de "foreignizing" (pour la traduction qui opère une "défamiliarisation »). Voir Lawrence Venuti, The Scandals of Translation: Towards an Ethics of Difference, New York : Routledge, 1998.

11. Mylène Lacroix, "Shakespeare au 'banquet' des langues étrang(èr)es », Actes des congrès de la Société française Shakespeare 31 (2014), 1-17, p.4, accessible en ligne: http:// 
shakespeare.revues.org/2793 (consulté le 10 février 2015). Cet article consacre de longs développements à Love's Labour's Lost.

12. Dans l'in-quarto de 1598 et l'in-folio de 1623, la lettre d'Armado à Jaquenetta (IV.i) est signée «Don Adriana de Armatho ».

13. Orthographié « Fortuna delaguar » dans l'in-folio de 1623.

14. Lynne Magnusson, «'To gase so much at the fine stranger': Armado and the Politics of English in Love's Labour's Lost ", in Shakespeare and the Cultures of Performance, éds. Paul Yachnin et Patricia Badir, Aldershot: Ashgate, 2008, 53-68, p. 67-68. Magnusson analyse l'hypothèse selon laquelle Armado aurait été inspiré lui aussi par un personnage historique, à savoir Don Antonio de Pérez, représentant de Philippe II d'Espagne à la Cour d'Élisabeth, hypothèse défendue par Gustav Ungerer (A Spaniard in Elizabethan England, The Correspondence of Antonio Perez's Exile, 2 vols. Londres : Tamesis Books Limited, 1974 et 1976; Anglo-Spanish Relations in Tudor Literature, 1956 ; New York : AMS Press, 1972, p. 81-154 et 189-211).

15. Voir Carroll in Shakespeare, Love's Labour's Lost, op. cit., p. 27.

16. W.C. Carroll, The Great Feast of Language in Love's Labour's Lost, Princeton: Princeton University Press, 1976, p. 53.

17. Voir Oxford English Dictionary, s.v. brawl n.1 (la rixe) et n.3 (la danse) et la définition de Randle Cotgrave dans son Dictionarie of the French and English tongues, Londres : Adam Islip, 1611, STC ( $2^{\mathrm{e}}$ éd.) 5830 : «A totter, swing, or swindge; a shake, shog, or shocke: a stirring, an uncertain and inconstant motion; [...] also, a brawle, or daunce, wherein many (men, and women) holding by the hands sometimes in a ring, and otherwise at length, moue all together " (s.v. Bransle). Consulté (via EEBO) le 10 février 2015.

18. Voir l'article de Chantal Schütz dans le présent recueil pour le commentaire de Thonoit Arbeau (Jean Tabourot) dans son Orchésographie sur le caractère «sauvage » de la canarie, danse espagnole très appréciée en France à la fin du XVIe siècle.

19. On peut également envisager l'altération phonétique de ces sons en «cocu ", autre jeu de mots polyglotte. Voir à ce sujet l'article de Chantal Schütz dans le présent recueil.

20. Patricia Parker, «Polyglot Punning in Shakespeare (and Others) » in Esthétiques de la nouveauté à la Renaissance, éds. François Laroque et Franck Lessay, Paris : Presses de la Sorbonne Nouvelle, 2001, 41-58, p. 51. À la suite de la glose d'A.L. Rowse, certains éditeurs de la pièce reformulent « haud credo » en " auld grey doe ». Sur la dimension «babélienne » du plurilinguisme comme pratique d'adaptation dans un monde d'après la Chute, voir l'article de Sophie Chiari dans le présent recueil.

21. Parker, op. cit., loc. cit.. Voir également son analyse de l'énigme de «l'envoi »: «a riddling ending in a "goose" (the French "oie" already contained within "envoi"), which may be incapable of any solution or answer, until we reflect that the Latin for "goose" is the word "anser" itself " (Ibid.).

22. William Shakespeare, Peines d'amour perdues, dans la traduction de :

Pierre Le Tourneur, in Shakespeare traduit de l'anglais, Paris : Mérigot, 1782 ;

François-Victor Hugo (1860), in Euvres complètes, éd. J-B Fort, Levallois-Perret: Cercle du bibliophile, 1967 ;

Émile Montégut, in Euvres complètes, Tome II, Paris : Hachette, 1867 ;

Pierre Messiaen, in Les Comédies, Paris : Desclée de Brouwer, 1939;

Jean-Louis Supervielle, in Euvres complètes éd. bilingue P. Leyris et H. Evans, Paris : Formes et Reflets, 1955 ;

Annie Berthet, éd. bilingue, Paris : les Belles Lettres, 1969 ;

Jean-Michel Déprats, Seyssel : Comp'act, 1990 ;

Jean Malaplate, in Comédies, éd. bilingue M. Grivelet et G. Monsarrat, Paris : R. Laffont, 2000.

23. Frances Yates voit dans Berowne/Brown une allusion à l'Italien Giordano Bruno (A Study of Love's Labour's Lost, Cambridge : CUP, 1936, chapitre 5, p. 89-101). 
24. William Shakespeare, Peines d'amour perdues, trad. Jean-Michel Déprats, in Euvres complètes, V. Comédies, éds. Jean-Michel Déprats et Gisèle Venet, Paris : Gallimard, Bibliothèque de la Pléiade, 2013, p. 1341, note 3.

25. Nathanaël est mentionné en tant que disciple de Jésus dans l'Évangile selon Jean (1.45-51). Dans la pièce de Shakespeare, on peut dire que ce personnage religieux («curate ») apparaît comme le « disciple » de Holofernes.

26. François Rabelais, Gargantua, chapitre XIII, in Euvres complètes, Tome I, Paris : Classiques Garnier, 1991, p. 60.

27. En réalité, Le Tourneur appelle Dull « Doll ».

28. Voir Samuel Johnson [1709-1784], «Preface to Shakespeare », in The Works of Samuel Johnson, Londres : Rivington et al., 1823, Volume 10, p. 149 : « a quibble was to him the fatal Cleopatra for which he lost the world, and was content to lose it. »

29. Shakespeare, trad. Le Tourneur, op. cit., p. 259.

30. Ibid., p. 240.

31. La non-traduction de ces trois noms est d'autant plus surprenante, même si l'on tient compte du peu d'intérêt que portait Le Tourneur aux jeux de mots, qu'il a traduit, dans le même volume, les noms de Froth («Écume ») et Elbow (« Le Coude ») dans Measure for Measure.

32. «Trogne " peut également faire penser à «trognon » et ainsi rappeler un fruit (je remercie Charlotte Coffin de m'avoir suggéré cette interprétation).

33. Le terme "moustique " apparaît en français au début du dix-septième siècle (voir l'entrée dans le Dictionnaire étymologique du CNRTL, accessible en ligne: http://www.cnrtl.fr/ etymologie/moustique, consulté le 10 février 2015). Depuis le début du vingtième siècle, il s'emploie pour désigner un petit enfant. Quant à Moucheron, qui a lui aussi ce sens figuré, il est attesté depuis le début du quatorzième siècle (Ibid., accessible ici: http://www.cnrtl.fr/ etymologie/moucheron, consulté le 10 février 2015).

34. À l'exception peut-être de « Phalène »...

35. Voir à ce sujet les remarques de Montégut sur ses propres choix : «Nous nous sommes abstenus jusqu'à présent de traduire les noms composés en assez grand nombre qui se rencontrent dans les comédies de Shakespeare, par des équivalents français qui puissent en rendre la signification généralement satirique. Ces noms composés ont un double rôle, ils nomment un personnage et ils peignent un caractère. Si on les traduit par des équivalents, ils continuent bien à désigner un caractère, mais ils cessent de désigner un personnage réel et ne désignent plus qu'un être de raison, une personnification morale. Cependant nous ferons une exception pour les personnages des pièces qui se passent en France, Peines d'amour perdues, Comme il vous plaira, Tout est bien qui finit bien, parce que les noms composés qui s'y rencontrent étant empruntés au vocabulaire de la langue anglaise, ne peuvent représenter des noms français. Jamais un paysan français ne s'est appelé costard, en revanche plus d'un paysan français s'est appelé et s'appelle Grossetête, ce qui est la traduction exacte du mot Costard » (op. cit., p. 411).

36. Son véritable nom n'est pas donné dans les textes qui le mentionnent.

37. Thomas Churchyard, "The phantasticall Monarkes Epitaphe", A pleasaunte laborinth, Londres : John Kyngston, 1580, STC (2 éd.) 5250, p. $7 \mathrm{r}^{\circ}-\mathrm{v}^{\circ}$ (orthographe modernisée par mes soins).

38. Nicolas Boileau, L'art poétique, III.129, in Euvres de M. Boileau Despréaux, Genève: Fabri \& Barrillot, 1716, Tome 1, p. 322 (accessible en ligne sur: http://gallica.bnf.fr/ark:/12148/ bpt6k5663056d/f361.image.r=, consulté le 10 février 2015).

39. Carroll rappelle aussi que l'adjectif «thrasonical " utilisé par Holofernes pour qualifier Armado (v.i.13) dérive du nom d'un personnage de l'Eunuque, pièce de l'auteur latin Térence, «the proper name once again having become descriptive of a class » (Carroll, Great Feast, p. 19).

40. C. Daniel Boughner, "Don Armado and the Commedia dell'Arte», Studies in Philology 37, 1940 : 201-224, p. 202-208. Sur la commedia dell'arte, voir notamment l'ouvrage de synthèse de Claude 
Bourqui, qui rappelle que « le Capitano n'est soldat qu'en paroles »; lui et les autres personnages types «évoluent à la surface de la réalité de référence qu'ils évoquent dans leurs propos » ( $\mathrm{L} a$ Commedia dell'arte, Paris : Armand Collin, 2011, p. 90). Sur l'influence de cette forme théâtrale sur Shakespeare, voir Kathleen Lea, Italian Popular Comedy, Oxford : Clarendon Press, 1934 ; Allardyce Nicoll, The World of Harlequin: A Critical Study of the Commedia dell' Arte, Cambridge : CUP, 1987 ; Frances K. Barasch, "Shakespeare and commedia dell'arte: an intertextual approach ", Shakespeare Yearbook 10, 1999 : 374-401 ; Michele Marrapodi (éd.), Italian Culture in the Drama of Shakespeare and His Contemporaries, Aldershot: Ashgate, 2007 ; la synthèse de Louise George Clubb, « Looking Back on Shakespeare and Italian Theater ", Renaissance Drama, New Series 36/37, Italy in the Drama of Europe, 2010 : 3-19.

41. Boughner, op. cit., p. 212.

42. Antoine Girard dit Tabarin [1584-1626], CEuvres complètes de Tabarin: avec les Rencontres, fantaisies et coq-à-l'âne facétieux du baron de Gratelard, et divers opuscules publiés séparément sous le nom ou à propos de Tabarin. Tome 2 / Le tout précédé d'une introduction et d'une bibliographie tabarinique par Gustave Aventin, Paris : P. Jannet, 1858, p. 141-142.

43. Voir à ce sujet mon article, «La Langue de Shakespeare, l'accent de Tony Harrison : Quand matter rime avec water », Actes des congrès de la Société française Shakespeare 31 (2014), accessible en ligne : http://shakespeare.revues.org/2803 (consulté le 10 février 2015).

44. Ainsi que le souligne Boughner à propos des prédécesseurs de Shakespeare dans l'adaptation de personnages stéréotypés de la comédie latine ou italienne sur les scènes anglaises, "Roister Doister may have furnished a prototype of the Terentian miles into whose making have gone English elements. Basilisco in Soliman and Perseda bears a name on the order of Armado, cultivates an erudite language full of inkhorn terms [...]. Again, Lyly's Tophas is a fusion of classical, Italian, and native materials shaped to satirize some outlandish aspirant who mimicked gentlemen and imitated the learned and elaborate forms of the Court. Shakespeare, however, succeeded far beyond his predecessors in the task of domesticating the braggart soldier on the Elizabethan stage » (op. cit., p. 220).

45. Christophe Camard, "'Be acquainted with my two zanies here': l'influence du théâtre professionnel italien sur le théâtre élisabéthain », Cahiers Shakespeare en devenir 8 (2014), Le texteItalie dans l'œuvre de Shakespeare, \$19, accessible en ligne: http://shakespeare.edel.univpoitiers.fr/index.php?id=737 (consulté le 10 février 2015).

46. Cité par Maurice Sand, Masques et bouffons (comédie italienne) : texte et dessins, Volume 1, Paris : Maurice Lévy, 1860, p. 199.

47. Voir aussi son allusion au « zany » Boyet (v.ii.463).

48. Gisèle Venet, Notice à la pièce dans Shakespeare, Comédies, op. cit,. p. 1336.

49. Carroll, op. cit., p. 20 sqq.

50. Boughner, op. cit., p. 217.

51. Voir Oxford English Dictionary, s.v. moth n.1, sens 3 et la citation du Polychronicon de Ranulf Higdon traduit par John Trevisa (antérieur à 1384), qui appelle les parasites de Cour « moths [L. tinea] » et « rattons ». 


\section{RÉSUMÉS}

Située en Navarre, l'action de Love's Labour's Lost met en contact des personnages français aux noms plus ou moins anglicisés (les nobles de France et de Navarre), un soldat espagnol fanfaron au nom évocateur de l'« Armada » réputée « invincible » et néanmoins défaite par les Anglais en 1588 (Don Adriano de Armado), des pédants aux noms tirés de la Bible (Holofernes et Nathaniel) et des personnages « secondaires » aux noms anglais renvoyant à des particularités physiques ou intellectuelles (Costard, Dull, Moth).

Dans une pièce où de nombreux jeux de mots reposent sur l'onomastique, il me semble que la réflexion sur les apparences qui se développe à travers l'échec des signes à révéler l'identité passe également par les noms propres. Afin de mieux comprendre la tension entre ethnocentrisme et exotisme qui structure cette pièce cosmopolite, j'étudie les différentes traductions des noms propres en français depuis la version de Pierre Le Tourneur (1776-1782) jusqu'à celle de Jean-Michel Déprats $(1990,2013)$ en passant par celles de Hugo (1859-1866), Montégut (1867-1873), Messiaen (1938-1939), Supervielle (1955), Berthet (1969) et Malaplate (2000).

Set in Navarre, Love's Labour's Lost brings together French characters whose names have been more or less accurately anglicised (the noblemen of Navarre and noblewomen of France), a Spanish braggart whose name is reminiscent of the supposedly "invincible" Armada that was scattered by the English fleet in 1588 (Don Adriano de Armado), a pair of pedants whose names originate in the Bible (Holofernes and Nathaniel) and "minor" characters with English names which refer to physical or intellectual features (Costard, Dull, Moth).

As many puns rely on onomastics, names serve to question the capacity of signs to express identity, thus fuelling the reflection on appearances and reality that is at the core of the play. In order to better understand the tension between ethnocentrism and exoticism that characterises this cosmopolitan play, I will study a few examples of how the characters' names have been translated into French over the centuries, from Pierre Le Tourneur's version (1776-1782) to JeanMichel Déprats's $(1990,2013)$ through translations by Hugo (1859-1866), Montégut (1867-1873), Messiaen (1938-1939), Supervielle (1955), Berthet (1969), and Malaplate (2000).

\section{INDEX}

Mots-clés : annomination, commedia dell'arte, jeu de mots, onomastique, Peines d'amour perdues, traductologie, traduction

Keywords : annominatio, commedia dell'arte, Love's Labour's Lost, pun, translation, translation studies

\section{AUTEUR}

\section{LAETITIA SANSONETTI}

Université Paris Ouest Nanterre La Défense 